\title{
Idoneidad del internista para el manejo óptimo del paciente diabético
}

\author{
Internists as the most appropriate physicians for optimal \\ management of the diabetic patient
}

\author{
Pedro Conthe \\ Jefe Sección de Medicina Interna. Hospital General Universitario Gregorio Marañón. Madrid
}

La Diabetes Mellitus (DM) es una de las enfermedades más comunes y con prevalencia creciente a nivel mundial, siendo los factores que más han contribuido a este incremento el envejecimiento poblacional, el sedentarismo y el aumento de la obesidad ${ }^{1}$. Es reconocido universalmente que la diabetes mellitus es una enfermedad metabólica en su origen y con criterios diagnósticos bien establecidos. Viene siendo considerada en los últimos años como una enfermedad eminentemente vascular en su expresión clínica, debido a alta morbimortalidad generada por la enfermedad coronaria y por la insuficiencia cardiaca. Debe ser reconocido sin embargo, que la DM se acompaña en su historia natural también de otros trastornos importantes que merman la calidad y expectativa de vida de los pacientes y que requieren, por ello, un óptimo abordaje temprano y una visión global del problema en toda su extensión ${ }^{1-5}$. Existen además en la DM (en la DM tipo 2 que supone cerca del $90 \%$ de los casos) unas situaciones previas en las que emerge el disturbio metabólico a las que posiblemente no se les da la importancia debida (intolerancia hidrocarbonada, glucemia basal alterada, síndrome metabólico...) y en donde enmascaradamente se va instaurando una lesión vascular progresiva, de forma que cuando se reconoce el diagnóstico de DM con los criterios actuales, en un gran número de pacientes (próximo al $50 \%$ ) ya existirán daños establecidos detectables de lesión macro y microvascular ${ }^{6,7}$. Su importancia radica no solo en el interés pronóstico de reconocer este hecho, sino también en la capacidad demostrada de mejorar los resultados a largo plazo, mediante una intervención precoz, basada en estilos de vida.

Las medidas preventivas son una de las mejores armas para disminuir la prevalencia y controlar a los individuos con alto riesgo de padecer DM, en los que puede obtenerse un impacto preventivo más beneficioso y eficiente que en estadíos ulteriores. Debemos aludir especialmente al binomio Obesidad-Sedentarismo (verdadero problema médico y sociosanitario del siglo XXI) que arrastra a la epidemia creciente de diabetes a nivel mundial, siendo reconocido que más del $80 \%$ de las personas con DM2 presentan ya sobrepeso u obesidad en el momento del diagnóstico y por cada kg de aumento de peso medio en la población, aumenta el riesgo de desarrollo anual de diabetes en un $5 \%$. El mal control metabólico en los diabéticos favorece a largo plazo el desarrollo de complicaciones, tanto microvasculares, afectando a arteriolas y capilares (nefropatía, retinopatía y neuropatía diabética), que comprometen gravemente la calidad de vida del paciente, como a los territorios macrovasculares afectando a arterias de gran y mediano calibre (vasculopatía periférica, enfermedad cerebrovascular y cardiopatía) incrementando seriamente la morbimortalidad. La Insuficiencia Cardiaca (IC) como síndrome final común de diversas cardiopatías es más frecuente en el paciente con DM tipo 2, de forma más precoz y con peor pronóstico ocasionando el mayor número de hospitalizaciones en el paciente mayor con $\mathrm{DM}^{9-11}$. Podemos afirmar que el tratamiento multifactorial y precoz de la DM dirigido a obtener el control de todos los factores de riesgo cardiovascular asociados al trastorno cardiometabólico (hipertensión arterial, tabaquismo, dislipemia, obesidad, etc.) retrasa el desarrollo de complicaciones, disminuye la mortalidad y mejora la esperanza y calidad de vida de estos pacientes. Aunque indudablemente la afectación vascular en toda su expresión protagoniza las complicaciones más prevalentes y mortales en la DM (en especial la enfermedad coronaria y la insuficiencia cardiaca, frecuentes motivos de hospitalización), no debemos olvidar que la DM es por encima de ello una enfermedad multiorgánica y sistémica, con afectación de distintos órganos (cerebro, riñón, ojos, sistema nervioso periférico, piel, sistema arterial periférico, sistema digestivo, sistema óseo y articular, entre otros). Las infecciones y las dermopatías en el paciente diabético merecerían capítulos aparte por su alta prevalencia y peculiaridades. Las alteraciones óseas y en metabolismo fosfocálcico / vitamina $D$ han sido destacadas y son complejas propiciando mayor riesgo de facturas por causas multifactoriales. Por ello, es evidente que estos pacientes requieren una visión global y un control multidisciplinar que permita detectar el problema precozmente y por supuesto tratar adecuadamente la diversidad de las complicaciones que se producen a lo largo de la historia natural de la enfermedad ${ }^{12}$.

Entre los principales valores y capacidades del internista debe ser reconocida su visión integradora y su polivalencia, facilitando la integración de los problemas médicos generalmente en un escenario habitual de pacientes pluripatológicos donde emerge la DM comúnmente, en su expresión más habitual, la DM tipo 2. Es sabido que la DM condiciona comparativamente un mayor número de hospitalizaciones y mayor duración de la estancia hospitalaria en comparación con la población no diabética, y además con incremento relativo de la mortalidad hospitalaria ${ }^{13}$. El porcentaje de ingresos con DM2 en los Servicios de Medicina Interna es superior, en diversos registros, al $30 \%{ }^{8}$. Por 


\section{Las medidas preventivas son una de las mejores armas para disminuir la prevalencia y controlar a los individuos con alto riesgo de padecer DM}

otra parte existe, como ya hemos comentado, una alta prevalencia de DM en la población anciana, base de la asistencia diaria del internista, donde la diabetes suele presentarse acompañada de complicaciones avanzadas que se potencian por la edad, existiendo mayor complejidad en el manejo farmacológico y mayor mortalidad cardiovascular y una mayor discapacidad en estos pacientes. Parece reconocerse hoy en día que la óptima asistencia al paciente con DM requiere una intercomunicación obligada entre los profesionales que asisten a los pacientes en distintos momentos de su enfermedad, integrando actuaciones y muy a menudo priorizando las medidas y los fármacos que más beneficio han demostrado y que son implantables en un paciente concreto, consiguiendo una adherencia terapeútica sostenible ${ }^{14}$, lo que entraña gran dificultad. El paciente diabético debe ser siempre candidato a una valoración por Medicina Interna. Junto con la historia clínica detallada y la exploración general metódica, rigurosa y propia del internista, en todo paciente se requiere una exploración específica de distintos aparatos y sistemas junto a un empleo razonado de técnicas básicas: analítica general y metabólica, metabolismo fosfocálcico, renal, hormonal y también marcadores biológicos inflamatorios de lesión vascular (PCR, microalbuminuria, etc.), electrocardiograma, fondo de ojo, y ocasionalmente técnicas más específicas: eco vascular, ITB (índice tobillo brazo), estudio neurofisiológico, etc.

Sin duda en el aspecto terapéutico, la valoración global implica también definir unos objetivos personalizados y aceptados por el paciente, junto con la imprescindible educación diabetológica, en los estilos de vida (reforzada y en seguimiento por enfermería), así como valoración de autocontroles y diversos parámetros, programando revisiones periódicas, en coordinación con Atención Primaria. Los objetivos del tratamiento de la DM2 deben conseguir controlar la hiperglucemia y sus síntomas, evitar las descompensaciones agudas, diagnosticar precozmente y retrasar las complicaciones crónicas y, en conjunto, mantener la calidad de vida del paciente ${ }^{3,5}$. Disponemos en la actualidad de diferentes tratamientos farmacológicos para el control de la hiperglucemia, cuyo uso debe ser escalonado y perfilado para obtener el máximo beneficio. La optimización del control glucémico supone un importante beneficio en términos de prevención de las complicaciones microvasculares y retrasar la afectación macrovascular a largo plazo. Los internistas deben tener el conocimiento, la experiencia y la implicación suficiente para un correcto manejo de toda la medicación antidiabética incluyendo distintos tipos y pautas de insulinización ${ }^{15}$. El control de la presión arterial según criterios actuales lo que habitualmente requiere combinación de fármacos, adaptados al perfil y tolerancia del paciente, muy en relación con los parámetros de función renal, y función ventricular. El control de la hiperlipidemia centrando el objetivo principal en el control del colesterol LDL debe ser considerado crucial y al menos tan importante como el control glucémico. La antiagregación del paciente diabético sin enfermedad establecida es un tema controvertido y requiere conocer los efectos colaterales y los riesgos hemorrágicos en un paciente concreto ${ }^{16}$. La elaboración de protocolos consensuados entre la Asistencia Primaria y la asistencia especializada para la mejor prevención y tratamiento continuado del paciente diabético deben ser compartidos por endocrinólogos, internistas y médicos de Atención Primaria.

Nadie puede dudar hoy en día de que el paciente con DM2 requiere de una visión integradora en el diagnóstico preciso de su enfermedad y de sus posibles complicaciones vasculares, no vasculares y en el planteamiento de la actuación terapéutica. Todo ello debe constituir una de las áreas prioritarias de la actividad clínica del internista del siglo XXI"17.

\section{Bibliografía}

1. Wilson PWF, Kannel WB. In: Hyperglycemia, Diabetes and Vascular Disease. Ruderman N et al, eds. Oxford; 1992

2. Soriguer F, Goday A, Bosch-Comas A, Bordiú E, Calle-Pascual A, Carmena R, et al. Prevalence of diabetes mellitus and impaired glucose regulation in Spain: the Di@bet.es Study. Diabetología 2012;55:88-93.

3. Estrategia en Diabetes del sistema nacional de Salud. Actualización 2012 http://www.sediabetes.org/Estrategia_en_diabetes_del_SNS_Accesible.pdf

4. Kotseva K, Wood D, De Backer G, De Bacquer D; EUROASPIRE Study Group. Cardiovascular prevention guidelines in daily practice: a comparison of EUROASPIRE I, II, and III surveys in eight European countries. Lancet 2009;373:929-40.

5. American Diabetes Association. Standards of medical care in diabetes 2012. Diabetes Care 2012;35 Suppl 1:S11-63.guias

6. Ascaso J. por el grupo de enfermedad Cardiovascular de la Sociedad Española de Diabetes. Diabetes Mellitus y enfermedad cardiovascular. Documento SED Ediciones Mayo Barcelona 2007

7. Diabetes Prevention Program Research Group Reduction in incidence of type 2 diabetes with lifestyle intervention or Metformine N Engl J Med 2002;346:393-403

8. Sánchez A, Sánchez M. El internista y la Diabetes. Rev Clin Esp 2008;208:115-7.

9. Nichols GA, Hillier TA, Erbey JR, Brown JB. Congestive heart failure in type 2 diabetes: prevalence, incidence, and risk factors. Diabetes Care. 2001;24(9):1614-9

10. Young LH, Wackers FJ, Chyun DA, Davey JA, Barrett EJ, Taillefer R, et al.; DIAD Investigators. Cardiac outcomes after screening for asymptomatic coronary artery disease in patients with type 2 diabetes: the DIAD study: a randomized controlled trial. JAMA 2009;301:1547-55.

11. Issa VS, Amaral AF, Cruz FD, Ayub-Ferreira SM, Guimaraes GV, Chizzola PR, et al. Glycemia and prognosis of patients with chronic heart failure-subanalysis of the Long-term Prospective Randomized Controlled Study Using Repetitive Education al Six Month Intervals and Monitoring for Adherence in Heart Failure Outpatients (REMADHE trial). Am Heart J 2010;159:90-7.

12. P. Gaede,P. Vedel,N. Larsen,G.V. Jensen,H.H. Parving,O.B. Pedersen Multi-factorial intervention and cardiovascular disease in patients with type 2 diabetes $\mathrm{N}$ Engl $\mathrm{J}$ Med 2003;348:383-93

13. Ryden L, Standall E, Bartnik M, Van den Berghe G, BetteridgeJ, de Boer MJ, et al. Guidelines on diabetes, pre-diabetes, and cardiovascular diseases: executive summary: The Task Force on Diabetes and Cardiovascular Diseases of the European Societyof Cardiology (ESC) and of the European Association for the Study of Diabetes (EASD). Eur Heart J 2007;28:88-136

14. Conthe $P$, Márquez Contreras $E$, Aliaga Pérez $A$, Barragán García B, Fernández de Cano Martín MN, González Jurado M, Ollero Baturone M, Pinto JL. Adherencia terapéutica en la enfermedad crónica: estado de la situación y perspectiva de futuro. Rev Clin Esp. 2014 May 7. pii: S0014-2565(14)00127-1

15. Pérez Pérez A, Conthe Gutiérrez P, Aguilar Diosdado M, Bertomeu,Martínez V, Galdos Anuncibay P, García de Casasola et al. Tratamiento de la hiperglucemia en el hospital. Medicina Clínica 2009, 132: 465-75

16. Ekström N, Cederholm J,Zethelius B, Eliasson B, Fhärm E, Rolandsson O, Miftaraj M, Svensson AM, Gudbjörnsdottir S. Aspirin treatment and risk of firstincident cardiovascular diseases in patients with type 2 diabetes: an observational study from the Swedish National Diabetes Register. BMJ Open. 2013 Apr 20;3(4). pii: e002688. doi: 10.1136/bmjopen-2013-002688. Print 2013

17. Tschöpe D, Hanefeld M, Meier JJ, Gitt AK, Halle M, Bramlage P, Schumm-Draeger PM. The role of co-morbidity in the selection of antidiabetic pharmacotherapy in type-2 diabetes. Cardiovasc Diabetol. 2013 Apr 10;12:62. doi: 10.1186/1475-2840-12-62. 